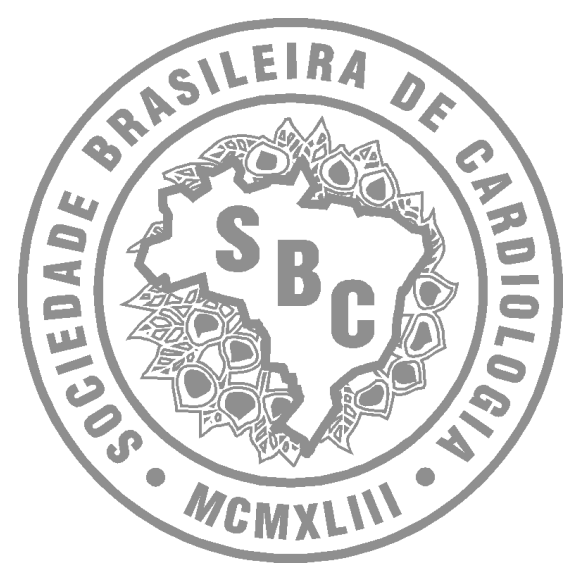

\title{
DIRETRIZ DE REABILITAÇÃO CARDÍACA
}

\author{
Editor \\ Ruy Silveira Moraes \\ Co-editor \\ Antonio Claudio Lucas da Nóbrega \\ Colaboradores \\ Renata Rodrigues Teixeira de Castro, \\ Carlos Eduardo Negrão, \\ Ricardo Stein, \\ Salvador Manoel Serra, \\ J osé Antônio Caldas Teixeira, \\ Tales de Carvalho, \\ Claudio Gil Soares de Araújo, \\ Maria J anieire Nazaré Nunes Alves
}




\section{Introdução}

Segundo a Organização Mundial da Saúde, reabilitação cardíaca é o somatório das atividades necessárias para garantir aos pacientes portadores de cardiopatia as melhores condições física, mental e social, de forma que eles consigam, pelo seu próprio esforço, reconquistar uma posição normal na comunidade e levar uma vida ativa e produtiva ${ }^{1}$. Há quatro décadas, quando esta definição foi estabelecida, os pacientes acometidos de infarto do miocárdio apresentavam grande perda da capacidade funcional, mesmo após serem submetidos ao tratamento daquela época, que implicava até 60 dias de repouso no leito. Por ocasião da alta hospitalar, os pacientes encontravam-se fisicamente mal condicionados, sem condições para retornar às suas atividades familiares, sociais e profissionais. Os programas de reabilitação cardíaca foram desenvolvidos com o propósito de trazer esses pacientes de volta às suas atividades diárias habituais, com ênfase na prática do exercício físico, acompanhada por ações educacionais voltadas para mudanças no estilo de vida. Atualmente, as novas técnicas terapêuticas permitem que a maioria dos pacientes tenha alta hospitalar precocemente após infarto, sem perder a capacidade funcional. Excluem-se desta condição os pacientes com comprometimento miocárdico grave e instabilidade hemodinâmica, distúrbios importantes do ritmo cardíaco, necessidade de cirurgia de revascularização miocárdica ou outras complicações não-cardíacas.

Nos últimos anos, foram descritos inúmeros benefícios do exercício regular para portadores de cardiopatia, além da melhora na capacidade funcional. A presente Diretriz abordará o papel da reabilitação cardíaca com especial ênfase no treinamento físico, ressaltando os seus efeitos cardiovasculares e metabólicos, os seus benefícios, indicações e contra-indicações. Em relação aos aspectos operacionais da reabilitação cardíaca, recomendamos a leitura da recente publicação da Sociedade Brasileira de Cardiologia "Normatização dos equipamentos e técnicas da reabilitação cardiovascular supervisionada" 2 .

\section{Abordagem multidisciplinar}

Além de dar ênfase à prática da atividade física, os programas de reabilitação cardíaca também envolvem outras ações desenvolvidas por profissionais das áreas de enfermagem, nutrição, assistência social e psicologia visando modificar outros aspectos que contribuem com a diminuição do risco cardíaco de forma global 3.4. O paciente e, eventualmente, membros de sua família, recebe informações sobre a fisiopatologia da doença cardíaca, os mecanismos de ação das drogas em uso, a relação da doença com a atividade física diária e as possíveis implicações na sua vida sexual e profissional. Quando necessário, os hábitos alimentares e aspectos nocivos do estilo de vida são reformulados, com especial ênfase na cessação do tabagismo. As intervenções psicológicas também devem ser consideradas, visando ao controle do estresse, o que pode ser obtido por meio de técnicas de relaxamento, terapia de grupo e tratamento da depressão.

Apesar de parecer muito lógico agregar uma abordagem mul432 tidisciplinar para melhorar o desempenho dos programas de reabi- litação que utilizam somente o exercício, uma recente revisão sistemática demonstrou maior redução das mortalidades total e cardíaca nos programas que utilizaram somente exercício físico, quando comparados aos que também agregaram a abordagem multidisciplinar ${ }^{5}$. A explicação para esta aparente contradição parece ser a grande diversidade de abordagens encontradas nos trabalhos publicados até o momento, dificultando a utilização da técnica de metanálise. Enquanto não houver confirmação desses resultados através de estudos melhor delineados, não devem ser abandonados os esforços para melhorar o estilo de vida dos pacientes, sempre que os recursos estiverem disponíveis ${ }^{6}(\mathrm{~A}, \mathrm{lla})$.

\section{Aspectos fisiológicos: adaptações ao treinamento físico}

A realização do exercício constitui um estresse fisiológico para o organismo em função do grande aumento da demanda energética em relação ao repouso, o que provoca grande liberação de calor e intensa modificação do ambiente químico muscular e sistêmico. Conseqüentemente, a exposição regular ao exercício ao longo do tempo (treinamento físico) promove um conjunto de adaptações morfológicas e funcionais que conferem maior capacidade ao organismo para responder ao estresse do exercício. Desta forma, após essas adaptações, um exercício de mesma intensidade absoluta (mesma velocidade e inclinação na esteira, por exemplo), provocaria menores efeitos agudos após um período de treinamento.

É importante destacar que os efeitos crônicos do exercício dependem, fundamentalmente, de uma adaptação periférica, que envolve tanto um melhor controle e distribuição do fluxo sangüíneo, como adaptações específicas da musculatura esquelética. Ocorrem modificações histoquímicas na musculatura treinada dependentes do tipo de treinamento, fazendo com que a atividade enzimática seja predominantemente oxidativa (aeróbica) ou glicolítica (anaeróbica lática).

Nesta seção, apresentaremos as adaptações típicas ao treinamento físico, lembrando que elas podem ser muito heterogêneas, dependendo não só das características do exercício a ser realizado, mas também do tipo de cardiopatia e da sua gravidade, da presença de outras condições médicas associadas e da capacidade funcional prévia do paciente.

Os dados que serão apresentados nesta seção são resultados de estudos clássicos que avaliaram processos fisiológicos. Em geral, são estudos experimentais presentes na grande maioria dos livros-texto de fisiologia do exercício. Sendo assim, essas informações apresentam nível de evidência B.

\section{Adaptações ao treinamento aeróbico}

Freqüência cardíaca - 0 treinamento aeróbico reduz tanto a freqüência cardíaca em repouso como durante o exercício realizado em cargas submáximas de trabalho. Esses efeitos parecem ser devidos à redução da hiperatividade simpática, aumento da atividade parassimpática, mudança no marca-passo cardíaco ou mesmo melhora da função sistólica. Apesar de o treinamento físico induzir melhora da potência aeróbica máxima, ele não modifica, 
de modo apreciável, a freqüência cardíaca máxima. Ou seja, pacientes treinados aerobicamente alcançarão a mesma freqüência cardíaca máxima de antes do treinamento, porém serão necessários níveis mais intensos de esforço para que essa freqüência cardíaca máxima seja alcançada ${ }^{7}$.

Pressão arterial - 0 treinamento físico reduz a pressão arterial de repouso e durante exercício submáximo ${ }^{8}$. Da mesma forma que ocorre com a freqüência cardíaca, o treinamento físico parece provocar pouca alteração na pressão arterial máxima aferida no pico do esforço.

Consumo de oxigênio - 0 consumo máximo de oxigênio $\left(\mathrm{VO}_{2}\right.$ máx) avalia de forma específica a capacidade aeróbica de um indivíduo. 0 sistema de transporte do oxigênio sofre uma adaptação favorável com o treinamento físico, que se exterioriza através de maiores valores de $\mathrm{VO}_{2}$ máx. $\mathrm{O}$ consumo de oxigênio é determinado pelo débito cardíaco e pela diferença arteriovenosa de oxigênio. 0 treinamento físico aumenta a diferença arteriovenosa de oxigênio através do aumento da volemia, da densidade capilar, do débito cardíaco e da extração periférica de oxigênio durante o exercício. Nos pacientes portadores de cardiopatia, o treinamento aumenta em $10 \%$ a $30 \%$ o $\mathrm{VO}_{2}$ máx, sendo este aumento mais evidente nos primeiros três meses de treinamento ${ }^{7}$. A melhora da potência aeróbica máxima costuma ser inversamente proporcional à capacidade física antes do treinamento, sendo os pacientes mais comprometidos os que, proporcionalmente, obtêm as melhorias mais significativas.

Função ventricular - Para uma mesma intensidade de esforço submáximo, o indivíduo treinado apresenta o mesmo débito cardíaco, porém às custas de freqüência cardíaca mais baixa e volume sistólico maior ${ }^{7}$. A maior extração periférica de oxigênio durante o exercício pode permitir que o indivíduo treinado atinja a mesma intensidade de exercício com menor débito cardíaco. Como a freqüência cardíaca no esforço máximo é semelhante no indivíduo treinado e no destreinado, o aumento do débito cardíaco ocorre devido a aumento no volume sistólico.

A maioria dos estudos mostra mínima ou nenhuma melhora da fração de ejeção do ventrículo esquerdo em resposta ao treinamento físico. Nos pacientes portadores de insuficiência cardíaca, a melhora da classe funcional obtida com o treinamento físico é secundária às adaptações periféricas ao exercício, não havendo correlação entre a fração de ejeção do ventrículo esquerdo em repouso e a capacidade funcional ${ }^{9,10}$.

Metabolismo - Com o treinamento físico, a musculatura esquelética desenvolve grandes adaptações na densidade capilar, na estrutura protéica miofibrilar e na sua composição enzimática. Isso resulta em maior eficiência na utilização de lipídios como substrato energético, retardando a utilização de glicogênio muscular, prolongando o tempo de exercício e aumentando a intensidade de esforço que pode ser sustentado.

Considerando as adaptações aqui apresentadas, podemos concluir que um indivíduo treinado aumenta o volume sistólico máximo, o débito cardíaco máximo e a tolerância à acidose muscular, permitindo atingir um $\mathrm{VO}_{2}$ máximo mais elevado. Desta forma, mesmo que o limiar anaeróbico (intensidade do esforço a partir da qual a produção do lactato muscular suplanta a capacidade do organismo em removê-lo) continue a ocorrer no mesmo percentual do esforço máximo, este ocorrerá durante um consumo absoluto de oxigênio mais elevado. Sendo assim, o desencadeamento de acidose ocorrerá em intensidade mais elevada de exercício. Com o treinamento aeróbico, o aumento do limiar anaeróbico pode ser proporcionalmente maior que os aumentos obtidos do $\mathrm{VO}_{2}$ máximo, caracterizando um aumento da tolerância ao exercício submáximo. Essas adaptações têm repercussões práticas, permitindo ao indivíduo treinado suportar cargas submáximas maiores por mais tempo, retardando o desenvolvimento de acidose e fadiga.

\section{Adaptações ao treinamento de força}

A maioria das atividades físicas que envolve contração muscular não é puramente dinâmica ou estática. Os dois tipos de contração produzem diferentes respostas hemodinâmicas, conforme será descrito a seguir. As atividades com componente estático envolvem movimentos de baixa repetição contra resistências elevadas, em que predominam contrações do tipo estáticas ou isométricas, nas quais se desenvolve tensão sem encurtamento do ventre muscular. Essa tensão muscular aumentada leva à restrição do fluxo sangüíneo muscular durante a contração, devido à compressão das arteríolas e capilares que perfundem o leito muscular, desencadeando resposta pressórica desproporcional ao consumo de oxigênio local ${ }^{11-13}$. A pressão arterial sobe bruscamente ao início de uma contração estática, quando esta tende a limitar o fluxo sangüíneo arterial, na tentativa de manter a pressão de perfusão para a musculatura em atividade. Essa elevação ocorre tanto na pressão arterial sistólica quanto na diastólica, resultando em maior póscarga e menor pré-carga por diminuição do retorno venoso ${ }^{11-13}$, sendo observadas pressões arteriais médias de até $320 / 250$ mmHg em contrações máximas dos membros inferiores. A marcada elevação da pressão diastólica é uma das principais diferenças fisiológicas entre estes dois tipos básicos de contração ${ }^{11-13}$.

Durante a contração isométrica, observa-se aumento da freqüência cardíaca, que varia de acordo com a massa muscular envolvida na contração, com a força voluntária máxima e com a duração da contração ${ }^{14}$. Esse aumento, que não costuma ultrapassar valores entre $62,7 \%$ e $85,2 \%$ da freqüência cardíaca atingida durante um teste de esforço máximo em esteira, é o responsável pela elevação do DC, já que o volume sistólico, em geral, não se eleva durante a contração isométrica, podendo, inclusive, diminuir. Durante a contração isométrica, o aumento da pressão arterial diastólica aumenta a perfusão coronariana durante a diástole, reduzindo os episódios de isquemia miocárdica durante esse tipo de treinamento. Uma revisão de 12 estudos sobre o uso do treinamento de força em programas de reabilitação cardíaca mostrou que, em portadores de doença arterial coronariana estável, já em treinamento aeróbico por pelo menos três meses, adicionar o treinamento de força (resistência muscular localizada) parece ser bastante seguro (A), promovendo melhora da força muscular e da endurance, sem desencadear episódios de isquemia miocárdica, anormalidades hemodinâmicas, arritmias ventriculares complexas ou outras complicações cardiovasculares ${ }^{15}$ (Ila).

A força muscular é fundamental para a saúde, para a manutenção de boa capacidade funcional e para atingir qualidade de vida satisfatória. Ela pode ser aumentada através de exercícios contra sobrecargas progressivas de trabalho com componente estático 
cada vez mais elevado (sem ultrapassar 50-60\% da força de contração voluntária máxima). Nos últimos anos, o treinamento complementar de força passou a fazer parte dos programas de reabilitação cardíaca, ajudando a melhorar a endurance muscular, a função cardiovascular, o metabolismo, os fatores de risco coronariano e o bem estar geral ${ }^{16}$. A tabela I resume estes benefícios.

Apesar de os mecanismos de melhora serem diferentes, tanto o treinamento aeróbico quanto o treinamento de força produzem efeitos favoráveis sobre a densidade mineral óssea, tolerância à glicose e a sensibilidade à insulina. Para o controle do peso corporal, o treinamento de força aumenta o gasto calórico através do aumento da massa muscular magra e do metabolismo basal ${ }^{16}$. Em indivíduos jovens, o treinamento de força eleva a resistência muscular, mas afeta pouco o $\mathrm{VO}_{2}$ máx. Em idosos, Vincent e cols. ${ }^{17}$ demonstraram aumento superior a $20 \%$ na capacidade aeróbica após o treinamento de força durante 24 semanas, provavelmente secundário à elevação da atividade das enzimas oxidativas e por diminuição da fraqueza da musculatura nos membros inferiores, permitindo o prolongamento do tempo de exercício.

\section{Limitações cardiovasculares ao exercício}

\section{Limitações fisiológicas}

O exercício físico caracteriza-se, fundamentalmente, pela contração muscular esquelética. A contração muscular é um processo ativo que requer energia para a movimentação das miofibrilas e pode chegar a aumentar, em muitas vezes, a demanda energética em repouso, que equivale a um consumo de oxigênio de 3,5 ml. $\mathrm{kg}^{-1} \cdot \mathrm{min}^{-1}$, ou 1 equivalente metabólico (MET).

Os mecanismos fisiológicos que limitam a capacidade funcional durante a realização de exercícios dinâmicos podem estar relacionados à intensidade ou à duração do exercício. Durante exercícios com cargas progressivas de trabalho, como no teste de esforço, a intensidade máxima de esforço é determinada por fatores de natureza hemodinâmica e metabólica muscular, responsáveis pela transferência de oxigênio do ar atmosférico para as células musculares. A intensidade máxima possível de exercício é definida pelo débito cardíaco e pelo volume sistólico máximo e pela concentração das

\begin{tabular}{|lc|}
\hline \multicolumn{2}{|c|}{ Tabela I - Adaptações ao Treinamento de Força } \\
\hline Variável & Resultado \\
\hline Densidade óssea & $\Uparrow \Uparrow$ \\
Força & $\Uparrow \Uparrow \Uparrow$ \\
Freqüência cardíaca em repouso & $\Leftrightarrow$ \\
HDL & $\Uparrow \Leftrightarrow$ \\
LDL & $\Downarrow \Leftrightarrow$ \\
Massa muscular & $\Uparrow \Uparrow$ \\
Metabolismo basal & $\Uparrow \Uparrow$ \\
Mudança da resposta insulínica & $\Downarrow \Downarrow$ \\
Nível de insulina basal & $\Downarrow$ \\
Percentual de gordura corporal & $\Downarrow$ \\
Pressão arterial diastólica em repouso & $\Downarrow \Leftrightarrow$ \\
Pressão arterial sistólica em repouso & $\Leftrightarrow$ \\
Sensibilidade à insulina & $\Uparrow \Uparrow$ \\
Tempo de endurance máximo e submáximo & $\Uparrow \Uparrow$ \\
VO máximo & $\Uparrow \Leftrightarrow$ \\
Volume sistólico basal e máximo & $\Leftrightarrow$ \\
\hline
\end{tabular}

enzimas oxidativas, particularmente aquelas que participam do ciclo de Krebs. Muito embora seja adequado considerar o consumo máximo de oxigênio como indicador da potência aeróbica máxima, a intensidade de esforço máximo atingida no teste de esforço depende também da potência anaeróbica, dependente da concentração de enzimas glicolíticas. Quando consideramos exercícios submáximos sustentados por longos períodos de tempo, a integridade dos mecanismos termorregulatórios e a depleção do glicogênio muscular adquirem maior importância na limitação do esforço. No treinamento de força, os mecanismos anaeróbicos da ressíntese de adenosina tri-fosfato (ATP) são mais importantes na limitação do esforço do que os aspectos hemodinâmicos. Desta forma, as funções anaeróbicas alática e lática são os principais determinantes da intensidade de esforço nos exercícios estáticos e resistidos. Além dos mecanismos metabólicos periféricos envolvendo a musculatura em atividade, a fadiga muscular também sofre grande influência da capacidade subjetiva de sustentar a contração, fazendo da motivação para o exercício outro determinante da capacidade funcional.

\section{Limitações relacionadas à presença de doenças cardiovasculares}

Grande parte dos pacientes com doença cardiovascular estabelecida refere diminuição da capacidade funcional, a qual se relaciona com redução no $\mathrm{VO}_{2}$ máximo obtido durante realização de teste ergométrico (B). Nesses pacientes, a capacidade de exercício é determinada pela complexa interação entre os sistemas cardiovascular, respiratório, metabólico e muscular, somada à modulação pelo sistema nervoso autônomo. Dessa forma, qualquer desequilíbrio nessa interação pode diminuir a capacidade funcional do indivíduo (B).

Nos pacientes portadores de insuficiência cardíaca, a perda da capacidade funcional é dependente de alterações centrais e periféricas. As alterações centrais são decorrentes da incapacidade desses pacientes em aumentar adequadamente o volume sistólico e a freqüência cardíaca, resultando em menor fração de ejeção e menor débito cardíaco. Nos portadores de cardiopatia isquêmica, o exercício também é limitado pelo eventual desencadeamento de isquemia miocárdica (B). Do ponto de vista periférico, a perda da capacidade funcional resulta da diminuição da capacidade oxidativa do músculo esquelético, da menor perfusão muscular, da presença de disfunção endotelial, favorecendo o aparecimento de acidose ainda nas fases iniciais do exercício. A intolerância ao exercício observada nos pacientes com função sistólica preservada e déficit diastólico pode ser explicada pela redução do débito cardíaco secundária à limitação ao enchimento ventricular, com conseqüente prejuízo do mecanismo de Frank Starling (B). Pacientes com insuficiência cardíaca e hiperatividade simpática apresentam maior intolerância ao exercício quando comparados a pacientes sem disautonomia (B).

Outro importante fator de limitação durante o exercício é a presença de sintomas. Pacientes portadores de cardiopatias referem mais sensação subjetiva de cansaço e de dispnéia quando comparados a indivíduos saudáveis de mesma idade e peso, (B). Muitos destes pacientes também apresentam atrofia e falta de condicionamento da musculatura respiratória. 


\section{Benefícios da reabilitação cardiovascular}

\section{Isquemia miocárdica}

Entre os benefícios fisiológicos proporcionados pelo exercício, em pacientes com doença arterial coronariana estável, incluemse a melhora da angina em repouso (I), a atenuação da gravidade da isquemia induzida pelo esforço (Ila), a melhora da capacidade funcional (I) e o controle de alguns dos fatores de risco para doença cardiovascular (Ila). A melhora da isquemia miocárdica resulta do aumento do volume sistólico $(A)$, da atenuação da taquicardia durante o exercício para cargas submáximas de esforço (B), da melhora na resposta vasodilatadora dependente do endotélio (B) e no aumento de perfusão na microcirculação coronariana (B). Esse último benefício deve-se, provavelmente, ao recrutamento de vasos colaterais durante o exercício, clinicamente evidenciado pela atenuação da depressão do segmento ST durante o exercício (Ila, B) ${ }^{18,19}$ e melhora na perfusão miocárdica observada durante a cintilografia com tálio (B) ${ }^{20}$. Além disso, o treinamento físico associado à dieta pobre em gorduras pode reduzir a progressão da placa aterosclerótica, após um ano de acompanhamento (Ila, B), ou até regredir a placa aterosclerótica em pacientes coronarianos que realizam atividade física com gasto calórico de $1784 \mathrm{kcal}^{7} \mathrm{a}$ $2200 \mathrm{kcal} / \mathrm{semana}$, após 6 anos de seguimento (B) ${ }^{20}$. A melhora da isquemia miocárdica com o treinamento pode elevar os limiares isquêmicos relativo (limiar isquêmico atingido com carga mais elevada) e absoluto (surgimento de isquemia miocárdica com duplo produto maior).

\section{Insuficiência cardíaca}

Em pacientes portadores de insuficiência cardíaca, o surgimento de fadiga muscular e dispnéia durante o esforço limita a execução das atividades diárias, reduzindo a qualidade de vida. Após um período de treinamento físico regular, ocorre melhora na relação ventilação/perfusão pulmonar, na atenuação da hiperativação de receptores musculares quimiossensíveis e melhora da função respiratória por fortalecimento da musculatura respiratória (B). Nesses pacientes, o treinamento ajuda a reverter a disfunção endotelial (B), aumenta o consumo de oxigênio de pico $(A)$ e a potência aeróbica máxima (B), melhora a capacidade oxidativa do músculo esquelético $(B)$ e reduz a exacerbação neuro-humoral (B). Devido a esses efeitos, o exercício físico regular foi incorporado às medidas não-farmacológicas para o tratamento da insuficiência cardíaca $(\mathrm{I}, \mathrm{B})$, resultando em redução da resposta ventilatória durante o esforço, melhora da qualidade de vida e do prognóstico $(\mathrm{I}, \mathrm{B})^{21,22}$.

\section{Dislipidemia}

A atividade física exerce uma ação favorável sobre o perfil lipídico (D), principalmente nos casos de hipertrigliceridemia, níveis diminuídos de HDL-colesterol e alterações nas subfrações do LDLcolesterol ${ }^{23}$ (B).

Triglicérides e HDL-colesterol - Uma única sessão de exercício pode diminuir os níveis de triglicérides e aumentar os níveis de $\mathrm{HDL}$-colesterol de forma fugaz (B), desaparecendo o efeito num período em torno de dois dias. Isso ressalta a importância da realização regular de exercício físico no combate às dislipidemias (B). Programas de treinamento físico com um gasto calórico semanal de 1200 a 2200 kcal são suficientes para provocar um efeito favorável nos níveis de lípides séricos (B). Mesmo com mudanças mínimas no peso corporal, quanto maior o gasto calórico semanal, maiores os benefícios para a lipemia ${ }^{23}(\mathrm{I}, \mathrm{B})$.

LDL-colesterol - 0 exercício não parece alterar os níveis plasmáticos de LDL-colesterol total, mas provoca uma diminuição das partículas pequenas e densas de LDL-colesterol e um aumento do seu tamanho médio (B). A carga de treinamento necessária para obter esse benefício equivale a um gasto calórico equivalente a $23 \mathrm{kcal} / \mathrm{kg}$ por semana, o que para uma pessoa com $75 \mathrm{~kg}$ equivaleria a um gasto semanal de $1700 \mathrm{kcal}$. A mudança nas partículas do LDL-colesterol provocada pelo exercício é independente de alterações nos valores do LDL-colesterol total ${ }^{22}$ (B).

\section{Hipertensão arterial}

Estudos epidemiológicos têm revelado uma associação entre o baixo nível de atividade física e a presença de hipertensão arterial. Por outro lado, grandes ensaios clínicos aleatórios e metanálises ${ }^{24}$ têm confirmado que o exercício físico regular pode reduzir os níveis pressóricos (A). Dessa forma, a partir dos "anos 1990", diversas diretrizes passaram a recomendar a prática de atividade física como meio de prevenção e tratamento da hipertensão arterial (I). Esse efeito hipotensor do exercício pode ser observado após uma única sessão aguda de exercício dinâmico, perdurando por até 24 horas (B) ${ }^{25}$. Resultados recentes de uma metanálise, envolvendo 53 estudos clínicos controlados, mostraram que 0 exercício aeróbio regular leva a uma redução de 4,9 e 3,7 mmHg nos níveis de pressão sistólica e diastólica de repouso, respectivamente. Esta redução é ainda mais dramática em indivíduos de etnia negra e asiática que apresentam redução da pressão arterial sistólica em torno de 10,9 e 6,2 mmHg, respectivamente, e da pressão arterial diastólica em torno de 3,2 e 6,6 mmHg, respectivamente ${ }^{24}$.

\section{Obesidade}

Embora a obesidade esteja relacionada a fatores genéticos, estudos comportamentais associam o crescimento do número de indivíduos obesos ao estilo de vida adotado pelo mundo moderno, incluindo como fator importante o estilo de vida sedentário (A). A obesidade está fortemente relacionada à prevalência de diabetes mellitus tipo II, hipertensão e doenças cardiovasculares, entre outras doenças ${ }^{26}(\mathrm{~A})$. Está bem estabelecido que o exercício físico regular tem efeitos favoráveis sobre as co-morbidades da obesidade, particularmente naquelas relacionadas às doenças cardiovasculares e ao diabetes mellitus Tipo II ${ }^{27}$ (B). Indivíduos com sobrepeso ou obesos que se mantêm ativos apresentam menores níveis de mortalidade quando comparados aos indivíduos com sobrepeso ou obesos que não se exercitam (B). 0 exercício aumenta o metabolismo basal e a oxidação de lipídios e glicose (B), aumenta a sensibilidade à insulina, favorecendo o tratamento da síndrome metabólica muitas vezes associada à obesidade ${ }^{28}$ (B). Portanto, dieta hipocalórica com baixo teor de gorduras associada ao exer- 
cício físico regular constitui a base do tratamento não-farmacológico para o controle das co-morbidades associadas ao sobrepeso e obesidade, com conseqüente diminuição dos riscos de doenças cardiovasculares ${ }^{29}(\mathrm{I}, \mathrm{D})$.

\section{Mortalidade cardiovascular}

Estudos epidemiológicos indicam que o estilo de vida sedentário associa-se a um risco duplamente elevado de doença arterial coronariana ${ }^{30}$ (I, B). Foi observada uma redução em torno de $20 \%$ a $25 \%$ no risco de morte nos pacientes pós-infarto do miocárdio que estavam em programa de reabilitação cardiovascular, quando comparados aos pacientes submetidos a tratamento convencional, não utilizando exercício (I, B). Em 1999, Belardinelli e colaboradores realizaram o primeiro ensaio clínico randomizado a demonstrar que a reabilitação cardíaca tem impacto sobre a mortalidade, como desfecho duro nesse subgrupo de pacientes ${ }^{31}$. Dos 99 sujeitos que participaram do estudo, os cinqüenta indivíduos randomizados para programa de exercício físico por 14 meses apresentaram redução na mortalidade por todas as causas (42\%), por causas cardíacas ( $22 \%$ ), além de diminuição consistente na taxa de re-internação hospitalar por insuficiência cardíaca (19\%), quando comparados aos 49 arrolados para o grupo controle (A). Tanto em pacientes portadores de cardiopatia, como em indivíduos saudáveis, observa-se uma forte associação entre baixa capacidade física e risco de morte ${ }^{32}(\mathrm{I}, \mathrm{B})$.

\section{Osteomusculares}

Além de melhorar o condicionamento aeróbio dos pacientes, os programas de reabilitação cardiovascular também desenvolvem a coordenação motora, aumentam a amplitude de movimentos, a flexibilidade, a resistência e a força muscular (I, B). Como resultado, pode melhorar o padrão de movimento, diminuir o gasto energético e reduzir os distúrbios músculo-esqueléticos, freqüentemente encontrados em pacientes com doenças cardiovasculares, melhorando muito o bem-estar e a qualidade de vida ${ }^{33}$ (II, B).

\section{Diabetes mellitus}

Indivíduos ativos apresentam diminuição dos fatores de risco para o desenvolvimento de diabetes mellitus ${ }^{34}(\mathrm{I}, \mathrm{A})$, sendo este risco diminuído em $32 \%$ quando o gasto calórico semanal é equivalente a $2.000 \mathrm{kcal}^{35}$ (B). Adicionalmente, a realização de 150 minutos de exercício por semana, associada à dieta, redução ponderal e controle de estresse, reduz em $53 \%$ o desenvolvimento de diabetes mellitus tipo 2 em indivíduos intolerantes à glicose ${ }^{36}$ (A). 0 treinamento físico melhora a sensibilidade à insulina e 0 controle glicêmico em diferentes populações, independentemente de sexo, idade e peso corporal ${ }^{37}$ (IB).

Em pacientes diabéticos tipo 2, a melhora na sensibilidade à insulina possibilita diminuição da dose ou até mesmo eliminação de hipoglicemiantes orais ${ }^{38}$ (B). Em pacientes diabéticos tipo 1 ou tipo 2 insulino-dependentes, a melhora na sensibilidade à insulina possibilita redução da dose e do número de aplicações de insulina (B). Essas adaptações, no entanto, podem ser perdidas caso o treinamento físico seja interrompido ${ }^{39}(\mathrm{~B})$.

\section{Tabagismo}

Resultados de estudos abordando a interferência do exercício físico sobre o hábito de fumar são unânimes em demonstrar que a abolição deste hábito não é significativa, isto é, apenas 3\% dos fumantes deixaram de fumar durante um programa de exercício físico ${ }^{40,41}$ (B). No entanto, estes mesmos estudos ressaltam a importância do treinamento físico em minimizar as implicações nutricionais atribuídas ao abandono do tabagismo, principalmente aquelas relacionadas aos riscos de aumento do peso.

\section{Aspectos psicossociais}

De uma forma geral, a prática regular de exercícios é responsável por mudanças nos estados de humor, tais como diminuição na fadiga e na raiva, e aumento no vigor, no estado de alerta e na energia. Essas mudanças positivas são maximizadas com exercícios prolongados e de baixa intensidade ${ }^{42}$. Em pacientes envolvidos em programas de reabilitação cardíaca, o treinamento físico relaciona-se à redução do estado de ansiedade, do nível de depressão, da instabilidade emocional, da ansiedade traço e dos vários sintomas de estresse (irritabilidade, hostilidade, tensão, comportamento tipo A) ${ }^{43}$. Em se tratando de pacientes acometidos de infarto do miocárdio, eles tendem a retornar com mais rapidez ao trabalho, mantendo a mesma qualificação profissional ${ }^{44}$.

\section{Indicações para reabilitação cardíaca}

\section{Insuficiência cardíaca}

Diversos estudos têm demonstrado que a reabilitação cardíaca melhora a qualidade de vida e a capacidade funcional. Em janeiro de 2004, foi publicada uma metanálise colaborativa denominada ExTraMATCH ${ }^{45}$. $\mathrm{O}$ objetivo primário desta publicação que reuniu os dados de nove ensaios clínicos randomizados (ECR) de grupos paralelos foi 0 de determinar 0 efeito do treinamento aeróbico por, pelo menos, oito semanas na sobrevida de pacientes com insuficiência cardíaca congestiva (ICC), decorrente de disfunção sistólica do ventrículo esquerdo. Oitocentos e um indivíduos de ambos os sexos compuseram a amostra, sendo 395 randomizados para o grupo exercício e 406 para o grupo controle. 0 desfecho principal foi mortalidade total e o desfecho secundário, a combinação de morte e internação por ICC. Os pacientes foram seguidos por 705 dias em média, ocorrendo 88 mortes no grupo exercício (22\%) e 105 no grupo controle (26\%). O hazard ratio (razão de azar) foi de 0,65 (IC 95\% 0,46-0,92). Foram verificadas 127 internações no grupo exercício versus 173 no controle (razão de azar de 0,72 com IC 95\% entre 0,56-0,93). Foi constatada uma clara evidência de que o treinamento físico supervisionado é seguro e que reduz tanto a mortalidade quanto as internações por descompensação da ICC, com um NNT de 17 para prevenir uma morte em dois anos (I, A). Muito recentemente, foi publicada uma revisão sistemática ${ }^{13}$ composta por 29 ensaios clínicos randomizados, que arrolou um total de 1126 pacientes com classe funcional NYHA II e III. Os autores concluíram que os programas de reabilitação cardíaca, mesmo aqueles de pequena duração, me- 
Ihoram a capacidade funcional em média em 2,16 ml. $\mathrm{kg}^{-1} \cdot \mathrm{min}^{-1} \mathrm{de}$ consumo máximo de oxigênio, aumento médio de 2 minutos e 38 segundos no tempo de exercício e de 41 metros na distância percorrida no teste de caminhada de seis minutos (I, A).

\section{Revascularização percutânea}

Hambrecht e colaboradores compararam um grupo de indivíduos com doença arterial coronariana submetido a um programa de exercício por 12 meses, associado com terapia farmacológica, com outro submetido à intervenção percutânea associada à terapia farmacológica. Os autores constataram que os pacientes do grupo exercício apresentaram uma sobrevida livre de eventos de $88 \%$, obtiveram um aumento na capacidade funcional de $16 \%$, além de um ganho custo-efetivo de aproximadamente 100\% (U\$ 3429 grupo exercício versus U\$ 6956 grupo intervenção percutânea) ${ }^{46}$ (Ila, A).

\section{Transplante cardíaco}

Programas de reabilitação cardíaca com duração de 8 a 12 meses podem aumentar em até $50 \%$ a capacidade funcional de pacientes submetidos a transplante cardíaco, através do desenvolvimento de adaptações centrais e periféricas que melhoram a extração periférica de oxigênio e 0 desempenho hemodinâmico ${ }^{47,48}$. Para esse grupo de pacientes, os programas formais de exercício parecem ser mais proveitosos do que somente a prática da atividade física domiciliar, pois, além de recuperarem a capacidade funcional, eles beneficiam-se do suporte educacional, nutricional e do apoio psicológico disponíveis em programas estruturados de reabilitação cardíaca $(\mathrm{I}, \mathrm{A}){ }^{49-51}$.

\section{Valvopatias}

Os pacientes que necessitam de reparo ou de troca valvar comumente se encontram em classe funcional III ou IV da NYHA, apresentando uma redução considerável da capacidade funcional máxima (4 METs ou menos). Nesses pacientes, as características hemodinâmicas e os sintomas referidos apresentam muita semeIhança com a dos pacientes portadores de insuficiência cardíaca ${ }^{52}$. Nos primeiros seis meses após serem submetidos a uma cirurgia valvar de sucesso, observa-se uma melhora importante na classe funcional e nos parâmetros hemodinâmicos, tanto em repouso quanto em exercício, os quais tendem a evoluir até 12 meses após a cirurgia. Os indivíduos submetidos ao treinamento físico após troca valvar apresentam melhora da capacidade funcional entre $19 \%$ e $38 \%$, quando comparados aos pacientes que não se exercitam ${ }^{53,54}(\mathrm{I}, \mathrm{A})$.

\section{Doença arterial coronariana}

Ao ingressarem em um programa de reabilitação cardíaca, os pacientes portadores de cardiopatia isquêmica podem esperar meIhora dos sintomas de angina (I), a atenuação da gravidade da isquemia induzida pelo esforço (Ila), a melhora da capacidade funcional (I) e melhor controle de diversos fatores de risco para doença cardiovascular (Ila). De acordo com as conclusões de recen- te metanálise, envolvendo 8940 pacientes oriundos de 48 estudos sobre o impacto da reabilitação cardíaca sobre a mortalidade, o exercício tende a reduzir em $20 \%$ a mortalidade por todas as causas e em $26 \%$ a mortalidade cardíaca (I, A) ${ }^{55}$.

\section{Estratificação de risco cardiovascular}

Antes de iniciar um programa de exercício físico para portadores de cardiopatia, é preciso estabelecer se o exercício pode representar algum risco para o paciente. Após a realização da anamnese e do exame físico, é fundamental a realização de um teste de esforço progressivo máximo para identificar o desencadeamento de isquemia miocárdica, disfunção ventricular, arritmias cardíacas e distúrbios da condução atrioventricular ${ }^{56}$. Este teste possibilita a determinação do $\mathrm{VO}_{2}$, da freqüência cardíaca e da pressão arterial sistólica de pico que também são importantes na avaliação do paciente cardiopata. Quando disponível, a avaliação pulmonar e metabólica através de análise dos gases expirados durante o teste de esforço deve ser realizada. Ela permite avaliar, com mais precisão, a capacidade funcional do paciente, em especial, o V0 máx e os limiares ventilatórios, as quais também poderão ser muito úteis na prescrição do exercício. A partir da anamnese, do exame físico e do teste de esforço, os pacientes deverão ser estratificados como sendo de risco baixo, moderado ou alto para iniciarem um programa de reabilitação cardíaca (tab. II). Os pacientes de baixo risco cardiovascular devem ser reavaliados a cada ano, enquanto aqueles classificados como de moderado a alto risco, devem ser avaliados mais precocemente (a cada seis meses ou sempre que ocorrer alguma modificação clínica) ${ }^{57-60}$.

\begin{tabular}{|ll|}
\hline Tabela II - Estratificação de risco para inclusão de pacientes em \\
programas de reabilitação cardíaca
\end{tabular}




\section{Contra-indicações à prática da atividade física}

Após a estratificação de risco inicial, os pacientes devem ser reavaliados no início de cada sessão de exercício, para a detecção de sinais e sintomas sugestivos de descompensação cardiovascular, que possam resultar em risco aumentado de complicações durante $o$ treinamento. Nos portadores de cardiopatia isquêmica, devese estar atento a mudanças no padrão de angina e, nos portadores de insuficiência cardíaca, o aumento do peso corporal adverte para a presença de congestão pulmonar. 0 comportamento da pressão arterial sistólica e da freqüência cardíaca em repouso e durante 0 esforço também devem ser monitorados ${ }^{58}$.

Em pacientes com infarto do miocárdio anterior extenso, um estudo recente mostrou um aumento no ventrículo esquerdo quando submetidos precocemente a um programa de exercício (3 dias), provavelmente por interferência no processo de remodelamento ventricular. Para esse grupo específico de pacientes, parece ser mais seguro aguardar, pelo menos, oito semanas após o evento agudo para iniciar o treinamento físico ${ }^{55}$. As contra-indicações absolutas ao exercício são mostradas no quadro ${ }^{57}$.

\section{Custo / Efetividade}

Por serem uma das mais importantes causas de morte e incapacidades física, social, psicológica e laborativa, as doenças cardiovasculares têm merecido a aplicação de elevados recursos financeiros, por vezes, incapazes de atender às necessidades globais diagnósticas e terapêuticas. Ao considerar um índice de aderência de $100 \%$ aos programas de reabilitação, foi possível estabelecer

\begin{tabular}{|l|}
\hline \multicolumn{1}{|c|}{ Quadro I - Contra-indicações absolutas à pratica de exercício físico } \\
\hline 1. Angina instável \\
2. Tromboflebite \\
3. Embolia recente \\
4. Infecção sistêmica aguda \\
5. Bloqueio AV de $3^{\circ}$ grau (sem marca-passo) \\
6. Pericardite ou miocardite aguda \\
7. Arritmia não-controlada \\
8. Insuficiência ou estenose mitral ou aórtica graves sem tratamento adequado \\
9. Insuficiência cardíaca descompensada \\
10. Hipertensão arterial descontrolada (PAS $\geq 200$ ou PAD $\geq 110$ ) \\
11. Depressão do segmento $S T>2$ mm \\
12. Problemas ortopédico ou neurológico graves \\
13. Diabetes mellitus descontrolada \\
14. Doença sistêmica aguda ou febre de origem desconhecida \\
15. Outros problemas metabólicos descompensados \\
\hline
\end{tabular}

a relação custo/efetividade, ou seja, o valor pecuniário investido para cada vida salva em um ano, levando-se em conta o gênero, a faixa etária, o tipo de supervisão e a presença ou não de doença cardiovascular (tab. III). Como referência, considera-se como excelência em custo/efetividade um determinado procedimento bem sucedido que resulta em um custo anual inferior a $\$ 20.000$ dólares norte-americanos. Entre $\$ 20.000$ e $\$ 40.000$ considera-se de acordo com as intervenções habituais. Acima de $\$ 40.000$ é indicativo de pobre relação custo/efetividade.

A análise dos dados apresentados na tabela III sugere que 0 treinamento com exercício físico como intervenção primária e secundária de doença cardiovascular é custo/efetivo, desde que seja considerada uma aderência adequada ao programa ${ }^{61,62}$. Com ausência de absenteísmo, um programa não supervisionado pode efetivamente salvar vidas e economizar custos. Um programa supervisionado, obviamente mais caro do que um sem supervisão, também é considerado altamente custo/efetivo para a grande maioria dos pacientes com doença cardiovascular. Mesmo levando-se em conta condições mais realistas em termos de aderência ao programa de exercícios, como valores entre $50 \%$ após um ano e $30 \%$ após 5 anos, persiste uma ótima relação custo/efetividade para homens com doença cardiovascular, tanto para os programas sem supervisão, como para os com supervisão. 0 benefício potencial do treinamento com exercício físico no que tange à expectativa de vida nas mulheres é menor que nos homens, considerando-se uma mesma faixa etária e mesma condição da doença. O exercício supervisionado somente é custo/efetivo para as mulheres na faixa etária entre 55 e 74 anos. 0 exercício não supervisionado possui excelente custo efetividade em todas as mulheres com doença cardiovascular e nas mais jovens sem doença cardiovascular.

O seguimento de pacientes portadores de insuficiência cardíaca em classe funcional II e III, durante 14 meses, revelou que 0 grupo de pacientes que foi submetido a um programa de exercícios teve um incremento na expectativa de vida de 1,82 ano, em relação aos que não se exercitaram. A intervenção mostrou excelente relação custo/efetividade, com um custo estimado em \$1.773 por vida salva ao ano ${ }^{63}$.

No Brasil, mesmo havendo grande disparidade entre os serviços de reabilitação cardíaca, no que se refere à disponibilidade de recursos materiais e de pessoal, o custo do serviço prestado é muito inferior ao dos serviços de reabilitação norte-americanos aqui usados como referência. Dessa forma, podemos inferir que a relação custo/efetividade dos nossos programas de reabilitação cardíaca seja muito mais favorável do que a dos norte-americanos. Conseqüentemente, deve ser amplamente difundida a importância da reabilitação cardíaca no tratamento eficiente dos pacientes

\begin{tabular}{|c|c|c|c|c|c|}
\hline & \multirow{2}{*}{$\begin{array}{l}\text { Idade } \\
\text { (anos) }\end{array}$} & \multicolumn{2}{|c|}{ Homens (Custo por VSA*) } & \multicolumn{2}{|c|}{ Mulheres (Custo por VSA) } \\
\hline & & Sem supervisão & Com supervisão & Sem supervisão & Com supervisão \\
\hline \multirow[t]{3}{*}{ Sem doença cardiovascular } & $35-45$ & $\$ 645$ & $\$ 22.566$ & $\$ 4.915$ & $\$ 42.296$ \\
\hline & $55-64$ & $\$ 2.517$ & $\$ 15.015$ & $\$ 1.583$ & $\$ 49.353$ \\
\hline & $65-74$ & $\$ 1.237$ & $\$ 20.544$ & $\$ 1.367$ & $\$ 60.283$ \\
\hline \multirow[t]{3}{*}{ Com doença cardiovascular } & $35-45$ & $\$ 356$ & $\$ 10.783$ & $\$ 3.154$ & $\$ 34.677$ \\
\hline & $55-64$ & $\$ 912$ & $\$ 5.871$ & $\$ 2.464$ & $\$ 8.084$ \\
\hline & $65-74$ & $\$ 777$ & $\$ 9.034$ & $\$ 2.464$ & $\$ 12.839$ \\
\hline
\end{tabular}


com doença cardiovascular e incentivada a proliferação de programas no território nacional.

\section{Conclusão}

Os pacientes que aderem a programas de reabilitação cardíaca apresentam inúmeras mudanças hemodinâmicas, metabólicas, miocárdicas, vasculares, alimentares e psicológicas que estão associadas ao melhor controle dos fatores de risco e à melhora da qualidade de vida. Nos pacientes portadores de cardiopatia isquêmica e de insuficiência cardíaca, a reabilitação cardíaca reduz as mortalidades cardiovascular e total. Somado a esses benefícios, os programas de reabilitação cardíaca, quando adequadamente conduzidos, são seguros e muito custo/efetivos, devendo ser oferecidos a todos os pacientes.

\section{Referências}

1. Brown RA. Rehabilitation of patients with cardiovascular diseases. Report of a WHO expert committee. World Health Organ Tech Rep Ser 1964; 270: 3-46.

2. Araújo CGS, Carvalho T, Castro CLB, et al. Normatização dos equipamentos e técnicas da reabilitação cardiovascular supervisionada. Arq Bras Cardiol 2004; 83: 448-52.

3. Canadian Association of Cardiac Rehabilitation. Canadian guidelines for cardiac rehabilitation and cardiovascular disease prevention. 1st ed. Winnipeg, 1999.

4. Stone JA, Cyr C, Friesen M, Kennedy-Symonds H, Stene R, Smilovitch M. Canadian guidelines for cardiac rehabilitation and atherosclerotic heart disease prevention: a summary. Can J Cardiol 2001; 17: 3B-30B.

5. J olliffe JA, Rees K, TaylorRS, Thompson D, Oldridge N, Ebrahim S. Exercise-based rehabilitation for coronary heart disease [Cochrane Methodology Review]. The Cochrane Library, Issue 4, 2003. Chichester, UK: I ohn Wiley \& Sons.

6. Fletcher GF, Balady GJ , Amsterdam EA, Chaitman B, Eckel R, Fleg J, et al. Exercise standards for testing and training: a statement for healthcare professionals from the American Heart Association. Circulation 2001; 104: 1694-740.

7. Froelicher VF, Myers J N. Exercise and the Heart. 30 edição. Philadelphia: WB Saunders, 2000

8. Pescatello LS, Franklin BA, Fagard R, Farquhar WB, Kelley GA, Ray CA; American College of Sports. Exercise and hypertension. Med Science Sport Exerc 2004; 36: 533-53.

9. Detry J, Rousseau M, Vandenbroucke G, Kusumi F, Brasser LA, Bruce RA. Increased arteriovenous oxygen difference after physical training in coronary heart disease. Circulation 1971; 44: 109-18

10. Piña IL, Apstein CS, Balady G], et al. Exercise and heart failure: A statement of the American Heart Association Committee on exercise, rehabilitation, and prevention Circulation 2003; 107: 1210-25.

11. Donald KW, Lind AR, MC Nicol SW, Humphreys PW, Taylor SH, Stauton HP. Cardiovascular response to sustained (static) contractions. Circulation Research 1967 $20 \& 21:$ S1-15

12. Mitchel J R, Wildenthal K. Static (isometric) exercise and the heart: physiological and clinical considerations. Ann Rev Med 1974: $25 \cdot 369-81$.

13. Tuttle WW, Horvath SM. Comparison of effect of static and dynamic work on blood pressure and heart rate. J Appl Physiol 1957; 10: 294-6.

14. Lewis SF, Snell PG, Taylor WF, et al. Role of muscle mass and mode of contraction in circulatory responses to exercise. J Appl Physiol 1985; 58: 146-51.

15. Feigengaum MS, Pollock ML. Strength training. Phys Sport Med 1997; 25: 44-64

16. Kraemer WJ , Adams K, Cafarelli E, et al. Progression models in resistance training for healthy adults: position stand. Med Sci Sports Exerc 2002; 34: 364-80.

17. Vincent KR, Braith RW, Feldman RA, Kallas HE, Lowenthal DT. Improved cardiorespiratory endurance following 6 months of resistance exercise in elderly men and women. Arch Intern Med 2002; 162: 673-8.

18. Traverse JH, Wang YL, Du R, et al. Coronary nitric oxide production in response to exercise and endothelium-dependent agonists. Circulation 2000; 101: 2526-31.

19. Ehsani AA, Heath GW, Hagberg J M, Sobel BE, Holloszy J O. Effects of 12 month of intense exercise training on ischemic ST-segment depression in patients with coronary artery disease. Circulation 1981; 64: 1116-24

20. Schuler G, Hambrecht R, Schlierf G, et al. Myocardial perfusion and regression of coronary artery disease in patients on a regimen of intensive physical exercise and low fat diet. I Am Coll Cardiol 1992; 19: 34-42.

21. Hambrecht R, Niebauer J, Fiehn E, et al. Physical training in patients with stable chronic heart failure: effects on cardiorespiratory fitness and ultrastructural abnormalities of leg muscles. J Am Coll Cardiol 1995; 25: 1239-49

22. Piepoli M, Clark AL, Volterrani M, Adamopoulos S, Sleight P, Coats AJ . Contribution of muscle afferents to the hemodynamic, autonomic, and ventilatory responses to exercise in patients with chronic heart failure: effects of physical training. Circulation 1996; 93: 940-52.

23. Lacour J R. Lipid metabolism and exercise. Rev Prat 2001; 51: S36-41.

24. Whelton SP, Chin A, Xin X, He J. Effect of aerobic exercise on blood pressure: A metaanalysis of randomized, controlled trials. Ann Intern Med 2002; 136: 493-503.

25. Brandao Rondon MU, Alves MJ , Braga AM, et tal. Postexercise blood pressure reduction in elderly hypertensive patients. J Am Coll Cardiol. 2002; 39: 676-82.
26. Lee CD, Blair SN, J ackson AS. Cardiorespiratory fitness, body composition, and all cause and cardiovascular disease mortality in men. Am J Clin Nutr 1999; 69 : 373-380.

27. Grundy SM, Blackburn G, Higgins M, Lauer R, Perri MG, Ryan D. Physical activity in the prevention and treatment of obesity and its comorbities. Med Sci Sports Exerc 1999; 31: S502-8.

28. Saris WHM. The role of exercise in the dietary treatment of obesity. Int J Obes 1993; 17 (suppl.1): S17-S21.

29. World Health Organization. Obesity: preventing and managing the global epidemic Report of a WHO consultation on obesity. Geneva: World Health Organization, 1998.

30. MillerTD, Balady GJ , Fletcher GF. Exercise and its role in the prevention and rehabilitation of cardiovascular disease. Ann Behav Med 1997; 19: 220-229.

31. Myers J. Exercise and cardiovascular health. Circulation 2003; 107: E2-E5.

32. Belardinelli R, Georgiou D, Cianci G, et al. Randomized controlled trial of long-term moderate exercise training in chronic heart failure: effects on functional capacity, quality of life, and clinical outcome. Circulation 1999; 99: 1173-82.

33. Kemmler W, Lauber D, Weineck J, Hensen J, Kalender W, Engelke K. Benefits of 2 years of intense exercise on bone density, physical fitness, and blood lipids in early postmenopausal osteopenic women: results of the Erlangen Fitness Osteoporosis Prevention Study (EFOPS). Arch Intern Med 2004; 164: 1084-91.

34. Wilmore J H, Green JS, Stanforth PR, et al. Relationship of changes in maximal and submaximal aerobic fitness to changes in cardiovascular disease and non-insulindependent diabetes mellitus risk factors with endurance training: the HERITAGE Family Study. Metabolism. 2001; 50: 1255-63.

35. Helmrich SP, Ragland DR, Leung RW, et al. Physical activity and reduced occurrence of non-insulin dependent diabetes mellitus. N Engl J Med 1991; 325: 147-152.

36. The Diabetes Prevention Program Research Group. Reduction in the incidence of type 2 diabetes with lifestyle intervention or metformin. N Engl J Med 2002; 346 393-403.

37. Kelley DE, Goodpaster BH. Effects of exercise on glucose homeostasis in type 2 diabetes mellitus. Med Sci Sports Exerc 2001; 33: S495-S501.

38. Fujinuma $H, A b e R$, Yamazaki $T$, et al. Effects of exercise training on doses of oral agents and insulin. Diabetes Care 1999; 22: 1754-55

39. Albright A, Franz M, Hornsby G, et al. American College of Sports Medicine position stand. Exercise and type 2 diabetes. Med Sci Sports Exerc 2000; 32: 1345-60.

40. Tzou W, Vitcenda M, M cBride P. Smoking status after cardiac events and participation in outpatient cardiac rehabilitation. J Cardiopulm Rehabil 2004; 24: 94-9.

41. Twardella D, Kupper-Nybelen J, Rothenbacher D, Hahmann H, Wusten B, Brenner $H$. Short-term benefit of smoking cessation in patients with coronary heart disease: estimates based on self-reported smoking data and serum nicotine measurements. Eur Heart] 2004; 25:2101-8.

42. Weinberg RS, Gould D. Fundamentos da Psicologia do Esporte e do Exercício. Porto Alegre: Artmed Editora, 2a ${ }^{\text {a }}$ ed, 2001

43. Physical activity and psychological benefits. http://www.issponline. org/pdf/physactstatement.PDF.

44. Dugmore LD, Tipson RJ, Phillips MH, et al. Changes in cardiorespiratory fitness, psychological wellbeing, quality of life, and vocational status following a 12 month cardiac exercise rehabilitation programme. Heart. 1999; 81: 359-66.

45. Piepoli MF, Davos C, Francis DP, et al. Exercise training meta-analysis of trials in patients with chronic heartfailure (ExTraM ATCH). BrMed] 2004. 24; 328(7433): 189

46. Hambrecht R, Walther C, Mobius-Winkler S, et al. Percutaneous coronary angioplasty compared with exercise training in patients with stable coronary artery disease: a randomized trial. Circulation 2004; 109: 1371-8.

47. Kavanagh T, Yacoub MH, Mertens DJ , KennedyJ, Campbell RB, Sawyer P. Cardiorespiratory responses to exercise training after orthotopic cardiac transplantation. Circulation 1988; 77: 162-71.

48. Brubaker PH, Berry MJ , Brozena SC, et al. Relationship of lactate and ventilatory thresholds in cardiac transplant patients. Med Sci Sports Exerc 1993; 25: 191-6.

49. Kobashigawa J A, Leaf DA, Lee N, et al. A controlled trial of exercise rehabilitation after heart transplantation. N Engl I Med 1999; 340: 272-7.

50. Braith RW, Welsch MA, Mills RM J r, Keller JW, Pollock ML. Resistance exercise prevents glucocorticoid-induced myopathy in heart transplant recipients. Med Sci Sports Exerc 1998; 30: 483-489. 
Diretriz de Reabilitação Cardíaca

51. Braith RW, Mills RM J r, WilcoxCS, Davis GL, Wood CE. Breakdown of blood pressure and body fluid homeostasis in heart transplant recipients. J Am Coll Cardiol 1996; $27: 375-83$.

52. Stewart K, Badenhop D, Brubaker PH, Keteyian SJ , King M. Cardiac rehabilitation following percutaneous revascularization, heart transplant, heart valve surgery, and for chronic heart failure. Chest 2003; 123: 2104-11.

53. Nakamura M, Chiba M, Ueshima K, et al. Effects of mitral and/or aortic valve replacement or repair on endothelium-dependent peripheral vasorelaxation and its relation to improvement in exercise capacity. Am J Cardiol 1996; 77: 98-102.

54. Habel-Verge C, Landry F, Desaulniers D, et al. Physical fitness improves after mitral valve replacement. Can Med Assoc J 1987; 136: 142-7.

55. Kubo N, Ohmura N, Nakada I, et al. Exercise at ventilatory threshold aggravates left ventricular remodeling in patients with extensive anterior acute myocardial infarction. Am Heart] 2004; 147: 113-20.

56. Fletcher GF, Balady G, Froelicher VF, Hartley LH, Haskell WL, Pollock ML. Exercise standards: a statement for healthcare professionals from the American Heart Association Writing Group. Circulation 1995; 91: 580-615.

57. Moraes RS, Ribeiro J P. Heart disease. In: Frontera WR, editor: Exercise in Rehabilitation Medicine. Champaign: Human Kinetics, 1999.
58. American College of Cardiology/American Heart Association Clinical Competence statement on stress testing: a report of the American College of Cardiology/American Heart Association/American College of Physicians - American Society of Internal Medicine Task Force on Clinical Competence. J Am Coll Cardiol. 2000; 36: 1441-53.

59. Zoghbi GJ , Sanderson B, Breland J , Adams C, Schumann C, Bittner V. Optimizing risk stratification in cardiac rehabilitation with inclusion of a comorbidity index. J Cardiopulm Rehabil. 2004; 24: 8-13.

60. Merz CN, Paul-Labrador M, Vongvanich P. Time to reevaluate risk stratification guidelines for medically supervised exercise training in patients with coronary artery disease. J AMA 2000; 283: 1476-8

61. Lovensteyn I, Coupal L, Zowall H, Grover AS. The cost-effectiveness of exercise training for the primary and secondary prevention of cardiovascular disease. J Cardiopulm Rehabil 2000; 20: 147-55.

62. Ades PA, Pashkow FJ . Nestor J R. Cost-effectiveness of cardiac rehabilitation after myocardial infarction. J Cardiopulm Rehabil 1997; 17: 222-31.

63. Georgiou D, Chen Y, Appadoo S, Belardinelli R, Greene R. Cost-effectiveness analysis at long-term moderate exercise training in chronic heart failure. Am J Cardiol 2001; 87: 984-8. 